\title{
Eficacia de la vacuna Sinopharm contra el COVID-19
}

\author{
Efficacy of the Sinopharm vaccine against COVID-19
}

\section{Comentado de:}

Al Kaabi N, et al. JAMA. Published online May 26, 2021. doi:10.1001/jama.2021.8565. PMID: $34037666^{1}$

\section{Objetivo}

Evaluar la eficacia y los eventos adversos de dos vacunas inactivadas contra el COVID-19.

\section{Diseño, lugar y pacientes}

Se realizó un análisis interino preespecificado de un ensayo clínico aleatorizado (ECA) de fase 3, doble ciego, en los Emiratos Árabes Unidos y Baréin, que comenzó en julio de 2020. Se utilizaron los datos recolectados hasta el 20 de diciembre de ese año para el análisis intermedio de eficacia y hasta el 31 del mismo mes, para los eventos adversos.

Se incluyeron pacientes adultos de 18 años de edad o mayores, sin antecedentes conocidos de COVID-19.

\section{Intervención}

Las vacunas se desarrollaron a partir del aislamiento de dos cepas de SARS-CoV-2 (WIV04 y HB02) de dos pacientes en el Hospital Jinyintan, Wuhan, China, las cuales se emplearon por separado para desarrollar sendas vacunas (denominadas vacunas WIV04 y HB02 en lo sucesivo).

Los participantes fueron asignados al azar para recibir una de las dos vacunas inactivadas: WIV04 (5 $\mu \mathrm{g} /$ dosis; $\mathrm{n}=13.459)$ o HB02 (4 $\mu \mathrm{g} /$ dosis; $\mathrm{n}=13.465$ ), o un hidróxido de aluminio (alumbre, grupo placebo; $n=13.458$ ). Todos recibieron dos inyecciones intramusculares con 21 días de diferencia.

\section{Medidas de desenlace principales}

El resultado primario fue la eficacia contra el COVID-19 sintomático confirmado por laboratorio 14 días después de la segunda dosis de la vacuna entre los participantes que no tenían evidencia virológica de infección por SARS-CoV-2 en el momento de la aleatorización. El resultado secundario fue la eficacia contra el COVID-19 grave. La incidencia de eventos y reacciones adversas se recopilaron entre los participantes que recibieron al menos una dosis.

\section{Resultados}

De los 40.382 participantes asignados al azar para recibir al menos una dosis de las dos vacunas o control solo de alumbre (edad media de 36,1 [desvío estándar 9,1] años; 32.261 [84,4\%] de sexo masculino), se incluyeron en el análisis primario de eficacia $38.206(94,6 \%)$ participantes que recibieron dos dosis, contribuyeron con al menos una medida de seguimiento después de día 14 luego de la segunda dosis, y tuvieron resultados negativos en la prueba de reacción en cadena de la polimerasa con transcriptasa inversa (RT-PCR) para el SARS-CoV-2 al momento del enrolamiento.

La mediana de duración del seguimiento fue 77 (rango 1 a 121) días. Los resultados principales se resumen en la Tabla 1.

Se produjeron dos casos graves de COVID-19 en el grupo placebo y ninguno en los grupos de vacuna. Se produjeron reacciones adversas 7 días después de cada inyección en $41,7 \%$ a $46,5 \%$ de los participantes en los tres grupos. Los eventos adversos graves fueron raros y similares en los tres grupos: WIV04 $=$ $64(0,5 \%) ;$ HB02 = $59(0,4 \%) ;$ placebo = $78(0,6 \%)$.

Tabla 1. Resultados principales de eficacia de las vacunas a virus SARS-CoV-2 inactivado evaluadas en este ensayo. Nota: IC: intervalo de confianza; NA: no aplica

\begin{tabular}{|c|c|c|c|}
\hline COVID-19 sintomático & Vacuna WIV04 & Vacuna HB02 & Placebo \\
\hline Incidencia acumulada, $\mathrm{n}$ & 26 & 21 & 95 \\
\hline Tasa de Incidencia, n cada 1000 personas-año (IC 95\%) & $12,1(8,3$ a 17,8$)$ & $9,8(6,4$ a 15,0$)$ & $44,7(36,6$ a 54,6$)$ \\
\hline Eficacia, \% (IC 95\%) & $72,8(58,1$ a 82,4$)$ & $78,1(64,8$ a 86,3$)$ & NA \\
\hline
\end{tabular}

\section{Conclusiones}

En este análisis intermedio preespecificado de un ensayo clínico aleatorizado, la aplicación en adultos de cualquiera de las dos vacunas de SARS-CoV-2 inactivadas redujo significativamente el riesgo de COVID-19 sintomático, mientras que los eventos adversos graves fueron raros. Queda pendiente completar la recolección de datos para el análisis final.
Fuente de financiamiento/Conflicto de interés de los autores: Varios autores reportaron haber recibido subsidios del Ministerio de Ciencia y Tecnología de la República Popular China (2020YFC0842100) durante la conducción de este estudio. Algunos autores son empleados de China National Biotec Group, otros de Wuhan Institute of Biological Products, y otros, de Beijing Institute of Biological Products —todas estas empresas que desarrollaron la vacuna y patrocinaron el ensayo. Varios autores tienen además solicitudes de patentes pendientes.

\section{Comentario}

Desde el inicio de la pandemia se han desarrollado diversas vacunas para combatir el SARS-CoV-2. La vacuna Sinopharm pertenece al grupo de vacunas inactivadas. Éstas se desarrollan en un cultivo del agente patógeno del cual se obtienen las partículas virales sin capacidad de infectar al huésped, y éstas partículas o fragmentos son las que se utilizan para exponer el sistema inmunológico con el objetivo de generar una respuesta protectora frente al virus. 
Una de sus principales ventajas es que puede ser almacenada en un refrigerador estándar, entre 2 y 8 grados Celsius de temperatura, al igual que la vacuna AstraZeneca. Esta característica determina que estas vacunas sean muy adecuadas para el uso en entornos de bajos recursos.

Dentro de las limitaciones del ensayo resumido mencionamos que no se incluyeron embarazadas ni menores de 18 años, por lo que no se dispone de datos de seguridad y eficacia en estos grupos. Los participantes fueron, en su mayoría, adultos jóvenes, sanos, principalmente residentes en Medio Oriente, por lo que el estudio no tiene poder suficiente para evaluar la eficacia de la intervención en otros grupos poblacionales, como por ej. pacientes con enfermedades crónicas, mujeres, adultos mayores, habitantes de otras regiones geográficas o en aquellos con infección previa por SARS-CoV-2. Además, sólo se presentaron dos casos severos de COVID-19 entre los participantes, lo que no permite obtener conclusiones sobre desenlaces más duros. El ensayo tampoco puede responder a la pregunta de si esta vacuna serviría para prevenir los casos de infección asintomática.

A principios de mayo de 2021, una de las vacunas de Sinopharm, BBIBP-CorV (correspondiente a la desarrollada a partir de la cepa WIV04 evaluada en esta investigación) fue aprobada por la OMS para su uso de emergencia en mayores de 18 años de edad, lo que posibilita su distribución a través de la iniciativa COVAX en países con escasos recursos para acceder a la vacunación contra el COVID-19². Más recientemente, fue autorizada por el gobierno de China para su uso de emergencia en niños entre 3 y 17 años $^{3}$.

En Argentina, esta vacuna fue aprobada por la Agencia Nacional de Medicamentos, Alimentos y Tecnología (ANMAT) 4 inicialmente para personas entre 18 y 60 años, autorización que luego se amplió para su uso en mayores de 60 años, y es una de las tres vacunas que están actualmente disponibles en el país ${ }^{5}$. En los últimos días se conocieron resultados preliminares del estudio de efectividad de Campaña Nacional de Vacunación que informaron una reducción de la mortalidad por COVID-19 en personas de 60 años o más en Argentina, indicando que la efectividad de la vacuna Sinopharm para este desenlace sería en promedio de $61,6 \%$ (IC $95 \% 55,9$ a 66,2) luego de la primera dosis y de $84 \%$ (IC $95 \% 77,9$ a 88 ) luego de la segunda 6 .

\section{Conclusiones de la comentadora}

Este ECA está en curso en la actualidad, y la recolección de datos continúa con el objetivo de refinar las estimaciones de eficacia a largo plazo, identificar los posibles eventos adversos, determinar el grado de protección que infiere la vacuna Sinopharm contra la enfermedad severa y la mortalidad, y establecer la durabilidad de la inmunidad que confiere a quienes la reciben. Los resultados disponibles hasta el momento son prometedores, incluyendo la posibilidad de su indicación en niños, pero falta aún más información sobre su eficacia y seguridad que esperamos sea esclarecida en próximas publicaciones.

Carolina Carrara [ Servicio de Medicina Familiar y Comunitaria, Hospital Italiano de Buenos Aires; Departamento de Medicina, Instituto Universitario Hospital Italiano de Buenos Aires. carolina.carrara@hospitalitaliano.org.ar ]

Carrara C Eficacia de la vacuna Sinopharm contra el COVID-19. Evid Actual Pract Ambul. 2021;24(3):e002138. Available from: https://dx.doi.org/10. 51987/EVIDENCIA.V24I3.6955. Comentado de: Al Kaabi N, Zhang Y, Xia S, et al. Effect of 2 Inactivated SARS-CoV-2 Vaccines on Symptomatic COVID19 Infection in Adults: A Randomized Clinical Trial. JAMA. Published online May 26, 2021. doi:10.1001/jama.2021.8565. PMID: 34037666

\section{Referencias}

1. Al-Kaabi N, Zhang Y, Xia S, et al. Effect of 2 Inactivated SARS-CoV-2 Vaccines on Symptomatic COVID-19 Infection in Adults: A Randomized Clinical Trial. JAMA. 2021; Available from: 10.1001/jama.2021.8565.

2. World Health Organization. WHO lists additional COVID-19 vaccine for emergency use and issues interim policy recommendations; 2021. Available from: https://www.who.int/news/item/07-05-2021-who-lists-additional-covid-19-vaccine-for-emergency-use-and-issues-interimpolicy-recommendations [Last access: 2021-07-05]

3. National Health Commision of the People's Republic of China. China approves emergency use of domestic vaccines for 3-17 age group; 2021. Available from: http://en.nhc.gov.cn/2021-06/15/c_83879.htm [Last access: 2021-07-05]

4. Argentina. Ministerio de Salud de la Nación. Resolución 688/2021; 2021. Available from: https://www.argentina.gob.ar/normativa/nacional/resoluci\% C3\%B3n-688-2021-347264/texto [Last access: 2021-07-05].

5. Argentina. Ministerio de Salud de la Nación. Vizzotti anunció la autorización de la vacuna Sinopharm para mayores de 60 años; 2021. Available from: https://www.argentina.gob.ar/noticias/vizzotti-anuncio-la-autorizacion-de-la-vacuna-sinopharm-para-mayores-de-60-anos [Last access: 2021-07-01].

6. Ministerio de Salud Argentina. Estudio Efectividad de Campaña Nacional de Vacunación en Reducción de la Mortalidad por COVID-19 en personas de 60 años y mayores. Argentina. Resultados preliminares; 2021. Available from: https://save.org.ar/wp-content/uploads/2021/07/Resultadospreliminares-Efectividad.pdf. 